
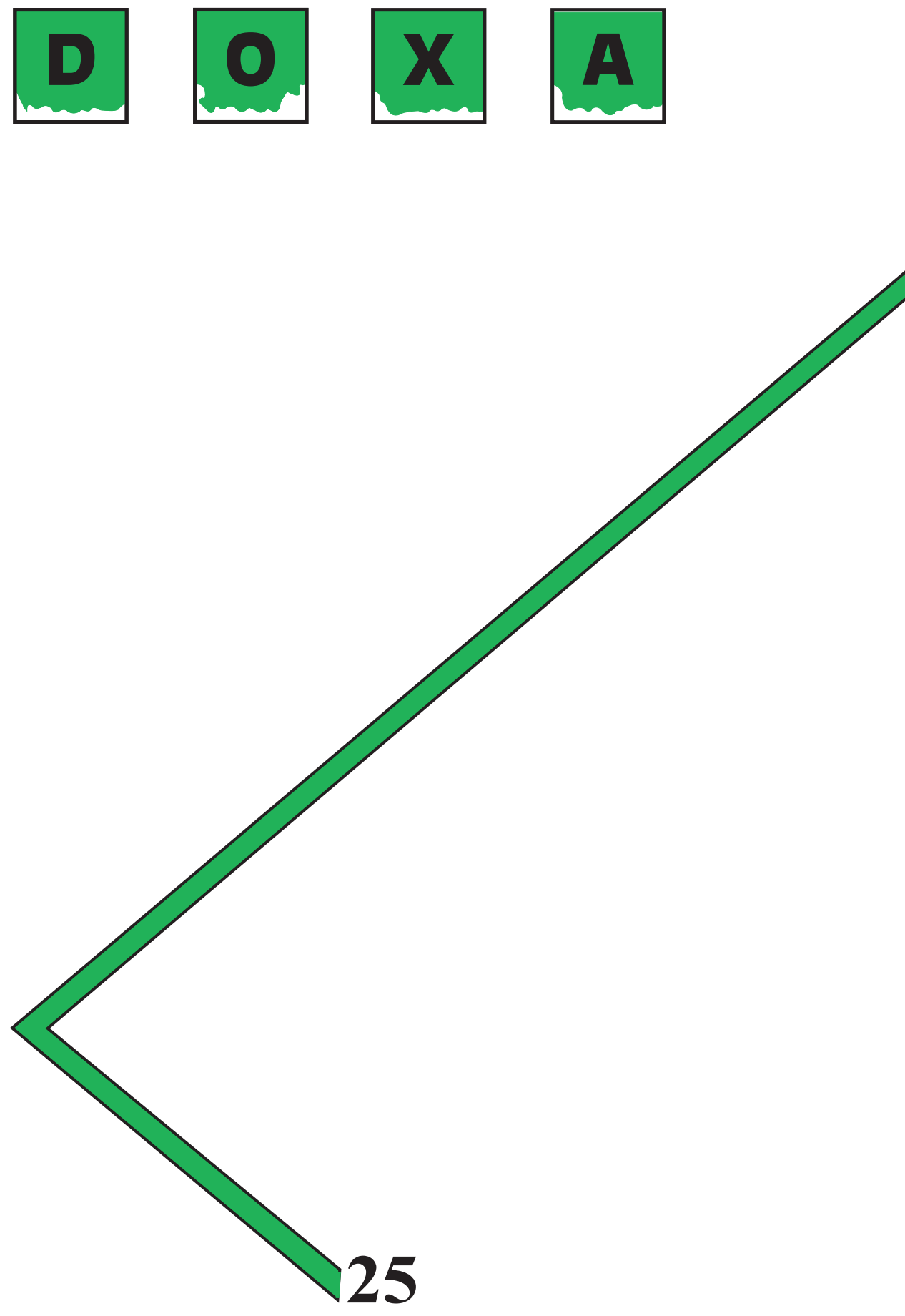

\title{
CUADERNOS DE FILOSOFÍA DEL DERECHO
}



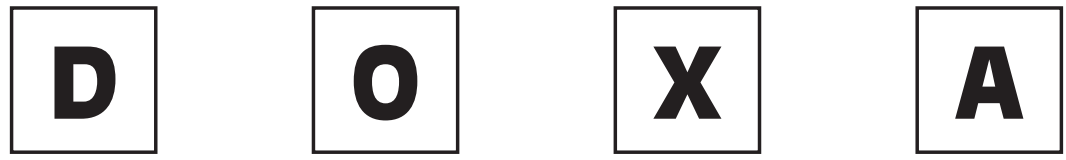

CUADERNOS DE

FILOSOFÍA DEL DERECHO

http: //cervantesvirtual.com/portal/doxa 
$\begin{array}{llllllllllll}\mathbf{P} & \mathbf{R} & \mathbf{E} & \mathbf{S} & \mathbf{E} & \mathbf{N} & \mathbf{T} & \mathbf{A} & \mathbf{C} & \mathbf{I} & \mathbf{O} & \mathbf{N}\end{array}$ 
lessandro Baratta fue un gran teórico social que se tomó en serio la famosa frase de Radbruch: "lo que necesitamos no es un mejor Derecho penal, sino algo mejor que el Derecho penal”. Paolo Becchi, en la nota dedicada a la memoria de Baratta con que se abre este número 25 de DOXA, presenta una biografía intelectual del que fue su maestro poniendo el acento tanto en sus aportaciones a la sociología del Derecho penal como a la filosofía del Derecho. En el primer campo, la influencia de Baratta, a través de su propuesta y elaboración de una criminología crítica y de un Derecho penal mínimo, ha sido verdaderamente enorme. Pero Baratta fue también un destacado filósofo del Derecho, y a la filosofía del Derecho volvió -como Becchi lo señala- en los últimos años de su vida, a través de la temática de los derechos humanos. Nadie que haya conocido a Sandro pudo sentirse indiferente ante su simpatía, su vitalidad, su inteligencia y su grado de compromiso en la lucha contra la injusticia.

La primera de las secciones monográficas del número, Problemas de filosofía moral y política, arranca con un trabajo de Martín D. Farrell dedicado a estudiar un importante aspecto de la obra de John Rawls, el gran filósofo de la moral y de la política desaparecido también en 2002. Las criticas que Farrell le dirige (en Rawls, el criterio maximin y la utilidad promedio) se pueden sintetizar en estas dos objeciones: 1) es cuestionable la suposición de Rawls de que en la posición originaria las partes aplicarían el criterio maximin para arribar a los principios de la justicia; 2) no está claro que el principio de diferencia, como piensa Rawls, sea superior al principio de la utilidad promedio. Farrell presenta su propia versión del principio de la utilidad promedio que, en su opinión, puede competir con éxito con el de la diferencia. Y aclara que su pretensión no es proporcionar 
un argumento decisivo en contra del principio de diferencia, sino "mostrar la debilidad de algunos argumentos de Rawls y realzar el atractivo del criterio utilitarista"

En La ética en la era de la técnica. Elementos para una crítica a Karl-Otto Apel y Hans Jonas, Paolo Becchi analiza críticamente otras dos importantes direcciones de la teoría ética en los últimos tiempos. En su opinión, Heidegger, "el más grande filósofo de nuestro siglo [o sea, del siglo XX]", habría hecho visible el peligro -y la génesis del mismo- que surge cuando -en la época moderna- la técnica no está ya subordinada a la naturaleza, sino que esta última es más bien "un objeto para someter a nuestro dominio”. La superación de Heidegger, el intento de mostrar el componente práctico de una filosofía de la técnica, habría que buscarlo en las obras de Apel y de Jonas. Sin embargo, Becchi considera que ambos intentos (entre sí muy distintos) son susceptibles de una misma objeción: Apel extiende el principio de la fundamentación filosófica al principio ético-normativo, pero su fundamentación intelectualista alcanzaría a justificar las reglas de habilidad o de prudencia, no las propiamente morales; y en la teoría de Jonas, la falta de distinción entre la praxis y la póiesis hace que no pueda alcanzar tampoco el nivel de la incondicionalidad del imperativo categórico, esto es, el de la obligatoriedad moral.

El trabajo de Rafael Alcácer Guirao, Prevención y garantías: conflicto y síntesis, comienza planteando lo que él llama "el dilema del Derecho penal": la pena se legitima porque protege la libertad de los ciudadanos, pero la imposición de la pena es una agresión a la libertad. Surge así un conflicto entre dos posibles fundamentaciones de la pena : una basada en la idea de prevención y que se corresponde con la tradición utilitarista de la ética que aspira a maximizar el bienestar de la colectividad y a justificar asi el limitar la libertad de individuos concretos; y otra que reposa en la idea de garantía, enmarcada en la moral kantiana que da prioridad a la libertad individual frente a las aspiraciones al bien común. Alcácer expone las diversas concepciones que, a propósito de ese conflicto, pueden encontrarse en el Derecho penal contemporáneo. Y concluye sosteniendo una concepción de la culpabilidad que responda a "una ponderación sintética de las dos direcciones enfrentadas: necesidad preventiva y garantía individual limitadora".

El trabajo de Antonio-Enrique Pérez Luño, Ciudadanía y definiciones, se dirige a precisar el significado y alcance de la noción de ciudadanía desde la perspectiva de una teoría de los derechos fundamentales. Con ese propósito esclarecedor, Pérez Luño construye un "mapa lingüístico" de las diversas definiciones lexicales de "ciudadania", y lleva a cabo también una indagación explicativa tendente a establecer el significado de ese concep- 
to desde una doble aproximación: sistemática e histórica. La conclusión a la que le lleva su análisis es la conveniencia de manejar una noción de "ciudadanía multilateral", entendida no sólo como "la posibilidad de ser titular simultáneamente de varias ciudadanias”, sino también como "la posibilidad de ejercerlas con mayor o menor intensidad según los sentimientos de cada ciudadano hacia cada una de estas comunidades políticas". En su opinión, esta definición explicativa no es una propuesta conceptual de contornos nebulosos, sino "un método integrador para la articulación de modalidades de ciudadanía que ya se hallan consolidadas”.

El artículo de Xacobe Bastida, Otra vuelta de tuerca. El patriotismo constitucional español y sus miserias se conecta en parte con el de Pérez Luño, pero el de Bastida pretende, como lo señala ya en la primera línea, "denunciar una impostura". Lo que considera impostura es el "patriotismo constitucional español" que cabe encontrar en el discurso político y académico en España y que, en su opinión, "no tiene nada que ver con el patriotismo constitucional en su formulación habermasiana”. La postura de Bastida es que "la construcción de Habermas bien pudiera tener acomodo en la realidad política -no en la jurídica-del Estado español, siempre y cuando se reconociese el derecho de autodeterminación, que es precisamente lo que se trata de evitar con el patriotismo de la constitución en su versión castiza”.

Finalmente, Rex Martín, en Obligación política: Algunos problemas y un intento de solución, aborda la cuestión de si existen razones justificadas para obedecer el Derecho. Martín critica a las teorías estándar que tratan de establecer "razones morales" para la obligación política y sólo consideran morales aquellas que "obligan a todas las personas en todo tiempo y lugar a obedecer todas las disposiciones jurídicas de su país”. En su opinión, ese enfoque debe sustituirse por otro basado en la noción de obligación especial en relación con las disposiciones jurídicas en cuanto tales. En las últimas páginas muestra un ejemplo de cómo funcionaría su propuesta en relación con un sistema político que contenga las nociones de derechos constitucionales fundamentales y procedimiento democrático .

La segunda sección monográfica, Sobre el positivismo jurídico, se abre con un artículo de Liborio Hierro, ¿Por qué ser positivista. Hierro caracteriza al positivismo jurídico a partir de tres tesis: la de las fuentes sociales del Derecho, la de la identificación objetiva del Derecho y la de la separación entre el Derecho y la moral, y considera que esta última constituye, en realidad, la base de las otras dos. La "gran virtualidad" de la tesis de la separación no estriba, en su opinión, "ni sólo ni principalmente, en la dudosa posibilidad de construir una Ciencia del Derecho meramente descriptiva y libre de valoraciones, sino en la posibilidad de mantener un punto de vista 
moral frente al Derecho positivo libre de determinaciones fácticas". La respuesta a la pregunta que da título a su artículo es que uno debe adherirse al "positivismo metodológico" por razones éticas (lo que diferencia de alguna manera su postura de la de Scarpelli quien, hace ya algunas décadas, sostuvo la adhesión ética al "positivismo como teoria").

La tesis de Hierro está estrechamente conectada con lo que defiende Tom Campbell en El sentido del positivismo jurídico. En opinión de este último, el positivismo jurídico no debe verse básicamente como una teoría analítica, descriptiva y explicativa del Derecho, sino más bien como una teoría normativa que trata de determinar "qué debe ser el Derecho, no respecto de su contenido, sino de su forma". El sentido del positivismo jurídico -del "positivismo ético"- consiste en considerarlo como "una teoría que recomienda que creamos y sostengamos sistemas jurídicos en los que el Derecho se identifique, se siga y aplique sin recurrir a las opiniones morales de aquellos envueltos en este proceso".

Luis Manuel Sánchez Fernández (Ironías, falacias, perversiones, enigmas. Sobre el positivismo y la tesis de la separación) parte de una caracterización del positivismo jurídico semejante a la de los dos anteriores autores, pero no adhiere a ningún tipo de positivismo jurídico, sino que, más bien, su trabajo está dirigido a señalar las críticas que el autor cree encontrar en esa concepción del Derecho. Afirma así que el positivismo incurre en la "falacia" de "pretender que se puede dar una imagen puramente descriptiva del Derecho"; que no constituye "por si mismo garantía de legalidad democrática ni menos de seguridad jurídica"; y que no tiene, "porque no es epistemológicamente apto para ello", una teoría de la argumentación judicial.

El modelo del mandato, el orden jurídico y las normas de competencia es el título del trabajo de Ulises Schmill que abre la sección de Artículos. Su idea central es la de reivindicar el modelo del mandato usado por Hobbes, Bentham y Austin, y criticado por Hart, como una manera adecuada de dar cuenta de las normas jurídicas y de los principales conceptos básicos del Derecho. Para ello, parte de una sencilla esquematización del mandato, que distingue entre el acto de mandar y el significado o "sentido normativo" de dicho acto, y reconstruye -con ayuda de un aparato formal y analizando las implicaturas (en el sentido de Grice) del modelo- los conceptos desarrollados por Kelsen en la nomodinámica y la nomoestática jurídica. Puesto que la principal crítica al modelo del mandato radica en la dificultad de dar cuenta de las normas de competencia, Schmill trata de reconstruir este tipo de reglas a través de las de mandato: según él, las normas de competencia son normas de mandato, de carácter facultativo, cuyo contenido es el acto de creación de otra norma (que pueden determinar parcial o completamen- 
te). A partir de ahí (de los conceptos de norma de competencia y, en particular, de facultad), analiza también las nociones de derecho fundamental, nulidad, derogación, nulificación y derrotabilidad.

En Dos conceptos de discrecionalidad jurídica, Isabel Lifante estudia los conceptos que subyacen a la caracterización usual del fenómeno de la discrecionalidad jurídica: libertad, indeterminación del Derecho y poder. En su opinión, cada uno de ellos puede ser entendido de dos maneras distintas y, si se combinan esos diversos sentidos, surgen dos conceptos muy distintos de "discrecionalidad jurídica". Mientras que el primero hace referencia a un efecto colateral de la existencia de normas, a un fenómeno periférico al ejercicio de la autoridad, el segundo apunta a un modo de conferir poderes para que los órganos jurídicos adopten decisiones atendiendo a las evaluaciones realizadas a la luz de las circunstancias de los casos concretos. Lifante considera que, en este último sentido, la discrecionalidad es un fenómeno central y necesario para llevar a cabo una de las funciones esenciales de los Derechos contemporáneos: la de promover activamente ciertos fines o valores.

María Inés Pazos toma como punto de partida de su trabajo (Derrotabilidad sin indeterminación) la noción de derrotabilidad que ya apuntó Hart en The Adscription of Responsibility and Rights. De acuerdo con esta noción, la derrotabilidad dependería de la existencia de significados respecto de los cuales no se pueden formular condiciones de aplicación necesarias y suficientes. Sin embargo, este sentido de derrotabilidad no debería confundirse con la indeterminación semántica (de ahi que el trabajo pretenda ser una crítica a lo que María Inés Pazos considera la concepción mas extendida de la derrotabilidad). Un enunciado sería derrotable cuando tiene la forma (o es equivalente a uno que tiene la forma) "Los A son B" y al mismo tiempo se cumplen dos condiciones: (1) Los A son normalmente $B$ y (2) existe al menos un hecho D que, cuando ocurre en conjunción con $A$, excluye B. Esta definición de derrotabilidad - a la que llama "genuina derrotabilidad"- no implica indeterminación sino que, por el contrario, presupone la determinación de las excepciones. La otra noción -más amplia-de derrotabilidad se refiere a los casos de enunciados y normas con excepciones indeterminadas.

¿De qué hablamos cuando hablamos de deberes jurídicos? (Algunas consideraciones sobre las fuentes del Derecho a partir de la tesis de Nino del caso especial) es el título de trabajo de Victoria Roca dedicado a estudiar las relaciones entre los conceptos de deber jurídico, pretensión justificativa del Derecho y fuentes del Derecho. Roca parte del análisis de Carlos Nino del concepto de validez jurídica y señala las insuficiencias en las que, a su juicio, incurren las reconstrucciones del concepto de deber jurídico que 
no consideran que la pretensión justificativa del Derecho sólo se satisface en la medida en que éste tenga una conexión con la moral. En su opinión, dado que la normatividad del Derecho sólo se comprende a partir de esa conexión, resulta plausible sostener-como hizo Radbruch-que las fuentes formales deben ser completadas con un cláusula que descalifique como normas jurídicas del sistema los casos de normas extremadamente injustas.

Tras haber criticado en diversos trabajos -varios de ellos publicados en DOXA- las corrientes ("ilusionistas") dominantes en la actual teoría del Derecho, Enrique Haba defiende en su nuevo artículo, Metodología realista-crítica y ética del razonamiento judicial (Realismo jurídico como una alternativa práctica al discurso normativista de los jueces) una alternativa de realismo crítico. Su propuesta se descompone en cuatro fases: 1) trazar el mapa de las interpretaciones posibles según los textos y que sea "de recibo" para la doctrina (momento lingüístico-dogmático); 2) reconstruir esas interpretaciones extrayendo su verdadera "gramática" y depurando sus defectos y "trampas": indeterminaciones, esencialismos y confusiones conceptuales, falacias implicitas, precomprensiones ocultas, sustrato ideológico, efecto emotivo... (momento analítico-crítico); 3) determinar (decidir) el objetivo práctico (fines y valores) del precepto (momento axiológico-teleológico); y 4) escoger la interpretación de hecho más eficaz para ese objetivo (momento empírico-instrumental). Según Haba, sólo esa metodología permite que el juez asuma su responsabilidad tanto intelectual como social y moral, pues el juez no puede lavarse las manos alegando que es el Derecho quien le impone la solución que dicta.

El Derecho como sujeto epistémico: hacia una epistemología constructivista del Derecho, de Gunther Teubner, es un intento de mostrar que la sociología del Derecho europea (en especial, teorías como las de Habermas, Foucault y Luhmann) puede ofrecer alternativas a la dicotomía tradicional entre realismo e idealismo epistemológico y entre individualismo y colectivismo metodológico. Teubner se refiere con ello a una reorientación radical de la teoría sociológica que reemplaza el realismo epistemológico por un constructivismo epistemológico, con lo que "los actores del individualismo metodológico son reemplazados por constructos como el discurso, la auto-reflexión y la auto-organización”. En el ámbito del Derecho, esta nueva perspectiva rechaza la "ingenua suposición realista de que los actores humanos, a través de sus acciones intencionales, configuran los elementos básicos de la sociedad". En su lugar, Teubner defiende tres tesis: 1) El Derecho no se refiere a una realidad social situada "ahi fuera", sino que construye su propia realidad social. 2) "[N]o son los seres humanos los que mediante sus acciones intencionales producen el Derecho como un artefacto cultural [sino que]... es el Derecho el que, como proceso comu- 
nicativo, crea actores humanos como artefactos semánticos a través de sus operaciones jurídicas". 3) Dado que la sociedad moderna se caracteriza por fragmentarse en diversas epistemes que se interfieren reciprocamente, el discurso jurídico se ve envuelto en una verdadera "trampa epistémica"; la existencia, de manera simultánea, de relaciones de dependencia e independencia con respecto a los otros discursos sociales es la razón por la cual "el Derecho moderno está permanentemente oscilando entre posiciones de autonomía y heteronomía cognitiva”.

Guillermo Lariguet, en Autonomía y unidad en el conocimiento jurídico, discute el papel de las teorías jurídicas en la delimitación de los casos y de las normas aplicables a los mismos. Se trata de qué manera afecta a la aplicación del Derecho cuestiones del tipo de cómo interpretar los términos y los conceptos de los supuestos de hecho de las normas o de si las condiciones de aplicación de una norma deben interpretarse como condiciones suficientes o necesarias. Para responder a estas cuestiones se formulan teorías jurídicas que tienen una importante relevancia práctica, pero que pueden entrar en conflicto entre sí (si surgen desacuerdos entre juristas). Con este planteamiento, Guillermo Lariguet se propone dos objetivos: estudiar las relaciones entre sistemas normativos y sistemas teóricos; y analizar la tensión que el recurso a estas teorías plantea entre la idea de autonomía y la de unidad tanto en el nivel conceptual como en el de los sistemas normativos. Su tesis sobre este último punto es que puede mostrarse que los conflictos posibles entre autonomía y unidad en el ámbito del conocimiento jurídico están fuertemente relacionados con los conflictos que se plantean en el ámbito de los sistemas normativos.

Finalmente, en Tiempo y contrato. Crítica del pacto fáustico, François Ost analiza la evolución de la institución del contrato desde el prisma de su relación con el tiempo (el contrato como un futuro anticipado y comprometido). El mito de Fausto, que vende su alma al diablo de una vez y para siempre, ilustraría en su extremo el paradigma clásico, formalista y voluntarista, del contrato: alienación al más fuerte (más que instrumento de colaboración), y atemporalidad, por su carácter a la vez instantáneo (la negociación previa es jurídicamente irrelevante, la clave es el acto del consentimiento) y eterno (inmutabilidad del contrato pese al cambio de circunstancias). Hoy, sin embargo, el contrato presenta una temporalidad más flexible, evolutiva y solidaria: relevancia de la formación progresiva del acuerdo, adaptación de la literalidad a cambios sobrevenidos, preservación de relaciones de confianza continuadas... Precisamente, frente al "tiempo fáustico", Ost defiende "un tiempo neguentrópico de alianza abierta y evolutiva entre compañeros; algo parecido a una buena fe compartida”. 
Cuestiones persistentes y microteorías es el título del trabajo de José Juan Moreso con el que se inicia la sección de Notas y que viene a ser la contribución del autor a una mesa redonda sobre "Los nuevos rumbos de la filosofía del Derecho". Entre las "cuestiones persistentes” de las que debe ocuparse la filosofía del Derecho, Moreso señala las que se enmarcan en la actual discusión que ha generado el positivismo hartiano. Pero llama la atención también sobre la importancia de elaborar teorías regionales -"microteorías"- que sirvan "como un banco de prueba de nuestros presupuestos más básicos". Y anima, en fin, a no "renunciar a la precisión y al rigor que ha alcanzado la filosofia analítica", aunque considera también como una obligación profesional la de "construir una filosofía del Derecho habitable para las preocupaciones que como seres humanos nos esperan”.

El trabajo que le sigue, De lo que no hay.-La inexistencia jurídica, constituye en cierto modo una aplicación de lo anterior. Ricardo Guarinoni parte de la importancia de un uso crítico del lenguaje, para poder dar cuenta de problemas como la discusión que tiene lugar entre los civilistas acerca de la "teoría del acto inexistente". Muestra así que la teoría sólo cobra sentido si se conecta con el concepto de normas que confieren poder. Señala las dificultades lógicas y filosóficas que acompañan a la noción de existencia. Pone de manifiesto el uso ideológico que los teóricos del Derecho hacen de esa figura (introducen elementos valorativos en forma pretendidamente descriptiva, y a partir de ahí infieren conclusiones teóricas y prácticas). Y concluye postulando la conveniencia de "describir los actos pretendidamente inexistentes como sujetos a nulidad, o no pertenecientes a la clase de que se trata".

Tomando como hilo conductor el comentario de un libro reciente de Alan H. Goldman, Andrea Greppi (Razones para seguir reglas. Observaciones sobre Practical Rules de A.H. Goldman) distingue las reglas en sentido estricto de otros tipos de normas y analiza con detenimiento la cuestión de si existen razones que justifican la adopción y el seguimiento de reglas. Greppi sostiene con énfasis la tesis de que el Derecho del mundo contemporáneo es básicamente un Derecho de reglas, esto es, el Derecho expresa el acuerdo sobre la inderogabilidad de algunas normas "que tienen que valer incluso a pesar de la aparición de razones nuevas" y fundamenta su afirmación, en último término, en la idea de que "no disponemos de ningún instrumento mejor que las reglas para poner freno al poder". Como se ve, un planteamiento enteramente afin al del positivismo jurídico defendido por Hierro o por Campbell en los trabajos que integran este mismo número de $D O X A$.

En Las comunidades primarias y el libre desarrollo de la personalidad, Rubén Ortega Cotarelo parte de una reciente obra de MacIntyre, Animales 
racionales y dependientes, para hacer una presentación de conjunto de la obra del filósofo estadounidense. Ortega Cotarelo resalta la impropiedad de etiquetarlo como un autor comunitarista: "lo que separa a MacIntyre de los liberales es tanto como lo que le separa de los comunitaristas". Y valora de manera muy positiva la filosofía de Macintyre, aunque no deja de dirigirle algunas críticas por el carácter "simplista" de su descripción de los Estados occidentales y su tendencia a centrarse exclusivamente en los aspectos negativos de los sistemas democráticos, lo que le impide "entrever todas sus virtualidades así como descubrir posibilidades ocultas de regeneración".

La reciente publicación de una serie de ensayos de otro autor estadounidense, Lon L. Fuller, es lo que motiva la nota de César Arjona que se publica a continuación. A diferencia de lo que suele ser más usual en los estudios sobre Fuller, Arjona (en Medios, fines y pluralidad de procesos en el pensamiento de Lon Fuller) se ocupa centralmente no de las relaciones del autor estadounidense con el positivismo analitico (el debate Hart-Fuller), sino con el instrumentalismo pragmático. Arjona destaca dos tesis que cabe encontrar en The Principles of Social Order: la relación entre los medios y los fines concebida como una relación dialéctica; y la existencia de una pluralidad de procesos para la consecución de fines sociales. E identifica tres aspectos que, en su opinión, constituyen la "herencia esencial" de Fuller: un uso sofisticado de la metodología instrumentalista; una ruptura de los límites de la juridicidad; y la idea de que el fin forma parte del mismo Derecho.

La sección de Notas se cierra con dos cartas de Carlos Cossio a Juan Ramón Capella, fechadas, respectivamente, en enero de 1972 y agosto de 1973. La primera es una (auto)biografía intelectual de Cossio destinada a proporcionar los materiales de una biografía del jurista argentino que Capella no llegó a escribir. La segunda tiene el interés de que en ella Cossio barrunta la tragedia que se cernía sobre Argentina.

La Entrevista que Miguel Ángel Rodilla le hace a José Delgado Pinto debería ser de lectura obligada para los filósofos del Derecho españoles, especialmente los más jóvenes. Dando pruebas de un admirable buen talante, un aquilatado sentido de la objetividad y un espíritu crítico y autocrítico que dice la verdad procurando al mismo tiempo no herir con ella, Delgado Pinto contesta a una serie de cuestiones que se organizan esencialmente en torno a tres bloques temáticos: los avatares de la filosofía del Derecho en España durante las cuatro últimas décadas; el papel del yusnaturalismo y del positivismo jurídico en la filosofía del Derecho; y la situación actual de nuestra disciplina, en el contexto español y en el internacional. No nos resistimos a recoger la recomendación que, al final de la entrevista, dirige 
"a quien se inicia en nuestra disciplina" (y que, al menos indirectamente, tiene también como destinatarios a los editores de una revista que ha publicado ya varios miles de páginas de material iusfilosófico): “que, en la medida en que se lo permita el sistema de promoción del profesorado, no publique demasiado".

Finalmente, la bibliografía sobre la filosofía del derecho en España en el año 2001 ha sido coordinada en esta ocasión por Hugo Ortiz. El lector podrá consultarla en http://cervantesvirtual.com/portal/doxa 